\title{
Review on Protocols and NDN Architecture for VANETs
}

\author{
Jugraj Singh \\ M.Tech Scholar, Department of Computer Science \\ \& Engineering, Global Institute of Management and \\ Emerging Technologies, Amritsar
}

\author{
Prabhdeep Singh \\ Assisstant Professor, Department of Computer \\ Science \& Engineering, Global Institute of \\ Management and Emerging Technologies, Amritsar
}

\begin{abstract}
The VANETs paradigm has become acquiring a great deal of fascination recently. VANETs provides brought on the breakthrough of varied innovative examine destinations, and the other unique position may be the road details community wherever an array of autos and also sources are generally included and also considerable portions of information are generally moved having large frequency. The important details firm cost to do business together with reduced debate effectiveness experienced from the road details community are generally demanding researchers to analyze more efficient together with scalable community architectures together with debate systems.
\end{abstract}

\section{Keywords}

VANETS, Fisheye state routing, Optimized Link State Routing Protocol, Ad hoc on-demand vector, TemporallyOrdered Routing Algorithm, NDN Architecture

\section{INTRODUCTION}

Vehicular Ad hoc Network is known as a unique kind of Mobile Ad hoc Network (MANET), which gets fascination from many researchers. In VANET each vehicle acts as a switch to change data between nodes in the system. It is made for vehicle-to-vehicle as well as infrastructure-to-vehicle communication. Such systems are employed in traffic control purposes, security purposes, driver aid and place based services. In VANETs energy use and storage capacity aren't confined and the particular having of your nodes could be resolved by using GPS. VANET has got unique capabilities like great movability together with the limitation certainly topology, truly minimal business transmission relation, unbounded multi-level measurement, facilities guide in which separate that by MANET. Coming from all these described capabilities, it is crystal clear in which standard MANET routingstandards has problems from locating secure routing trails in VANET environments. Therefore, more and more scientists have concentrated on proposing acceptable routing standards to manage with the very active character of VANET.

\section{VARIOUS ROUTING PROTOCOL 2.1 Fisheye state routing (FSR):}

It is comparable to url state routing process (LSR). Each node retains a topology table on the cornerstone of the latest information obtained from area nodes. It employs various change time for various articles in routing table to decrease how large control communications in big networks. The problem in FSR routing, is how big the routing table increases with upsurge inside network size. Alternative having might crash if the venue node is placed outside of variety of source node. The item link between excessive flexibility inside VANET, path to remote control location diminish accurate.

\subsection{Optimized Link State Routing Protocol (OLSR):}

It is definitely an optimization of an all-natural url state method for portable marketing hoc networks. Each node in the system decides some nearest nodes termed as multipoint relays which retransmits their packets. The nearest nodes that aren't within their MPR collection may only just study and technique the packet. That method decreases the total amount of retransmissions in a carried procedure.

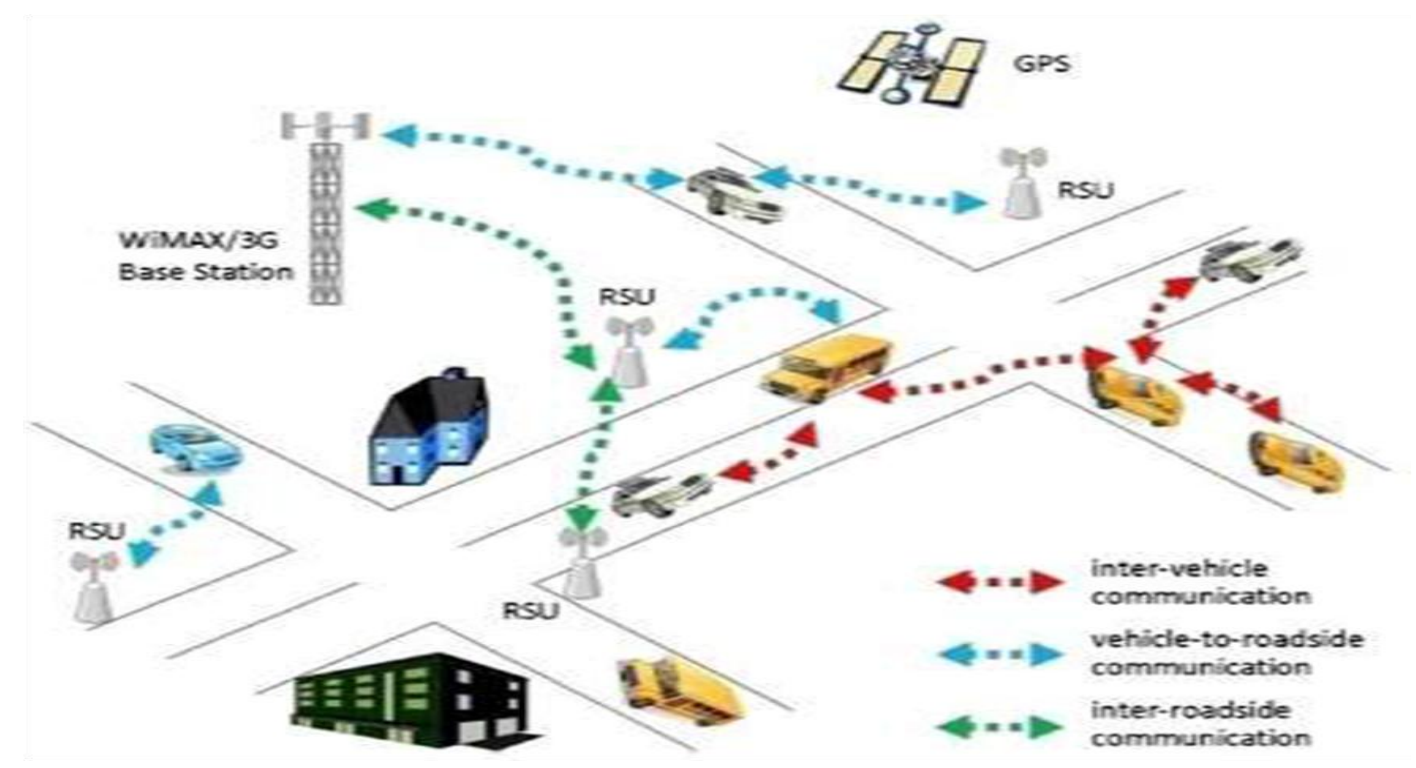




\subsection{Ad hoc on-demand vector (AODV):}

Can be a resource initiated routing process and employs HELLO communications to acknowledge their neighbors. Source node broadcasts a way request to their neighbors which fill ahead to the destination. Then the location unicast a way reply supply to the sender. Every node retains broadcastid which batches for new RREQ. Whenever a RREQ comes at a node, it checks the transmitted id if it's significantly less than or similar to prior information then it'll discard the packet.

\subsection{Temporally-OrderedRouting Algorithm:}

Every node builds a focused cyclic chart by transmission question packets. On receiving an issue supply, if the node features a road to location it'll send a answer supply, otherwise it leaves the packet. A node on receiving an answer supply can upgrade their height only when the height of supply is minimum than other reply packets. It provides a way to any or all the nodes in the network, though the preservation on most these paths are difficult in VANET.

\subsection{Greedy Perimeter Stateless Routing:}

Every node sporadically transmits a beacon message to any or all its nearest nodes comprising its id and position. If any node doesn't receives any beacon meaning from a friend for a particular timeframe then GPSR switch thinks that the buddy has failed or out of collection and removes the friend from its table. It takes greedy forwarding decisions applying information regarding immediate neighbors in the network. For just about any node if greedy forwarding is impossible then it employs perimeter of the location technique to acquire the following forwarding hop. In an area situation greedy forwarding is generally restricted since direct communications between nodes mightn't exist due to obstacles such as for instance for instance buildings and trees. Converting system topology in to planarized graph when greedy forwarding is difficult will weaken the efficiency of routing.

\subsection{Vertex-Based predictive Greedy Routing:}

This can be a multi-hop vehicle-to-infrastructure directionfinding way for down-town environment. This estimates a number of acceptable junctions through the basis node for you to mended structure and after that, exchange concept towards mended structure from the compilation of junctions. This utilizes spot, swiftness in addition to direction connected with automobiles regarding working out each compilation of appropriate junctions in addition to selfish forwarding. Inside computation connected with a number of appropriate junctions, an origin node ascertains the particular means among by itself as well as best mended structure with aid from navigation system. If the starting node gets a few path to the repaired infrastructure with same volume of junctions then it randomly selects one of the ways among them. It employs predictive on the web selfish redirecting to ahead data from source node to the closest repaired infrastructure.Each car keeps a desk, comprising id, place, speed and direction of its two-hop neighbors. The desk is sporadically up-to-date by trading beacon communications among nearest vehicles. With aid from a desk, the source node determines the weighted report for itself, current supply company and for two-hop neighbors. If a friend has the more expensive report than the present supply company, the source car forwards the supply to the friend usually, the present supply company holds the supply until it sees its friend that has higher weighted report than itself. VGPR have less control expense, reduces supply retransmissions, raises dependability of supply delivery, and reduces end to end delay.

\subsection{Roadside-Aided Routing:}

Is a design for effective redirecting in vehicular hybrid systems as opposed to cement redirecting protocol? Here highways are split directly into groups by applying path side models (RSUs), and the way includes vehicles and RSUs. These protocols aren't capable in large way scenarios as they may require fixed node or RSU.

\section{NDN ARCHITECTURE}

Connection inside NDN needs through items i.e., files consumers, through the exchange of two types of bins: Enchantment plus Facts . Similarly kinds of bins keep a identify which identifies a piece of data that can be transmitted in one particular Facts packet. Anyone parts this identify of your excellent minimal files directly into a good Enchantment discipline plus produces the item to your network. Modems employ this identify in order to in front this Enchantment when it comes to the feeling producer(s). Following a Enchantment actually reaches any node which is the important files, this node might reunite any Facts discipline which contains both the name and the fabric, together with any brand through the producer's important which usually holds each .This Facts discipline utilizes in the opposite direction this move utilized through the Enchantment to go back to this looking for consumer. To carry out the Interest and Data packet sending extends, just about every NDN transform retains some files constructions: any Pending Enchantment Table (PIT), any Forwarding Facts Base (FIB), and also a Content Store (CS), along with a Forwarding Process component that makes certain whether or not, where and when you must in front just about every Interest packet.

\section{RELATED WORK}

Leng, Supeng et al. offered a comprehensive evaluation of various medium accessibility get a grip on systems in vehicular offer hoc sites, which can be needed for the applications of both path security and the ease of driving. The part first discusses particular demands and issues for MAC design in VANETs and introduces the appropriate common as fundamental information for research. Furthermore, some essential dilemmas and probable solutions are addressed by the evaluation of current papers and the presentation of works, including multichannel accessibility get a grip on, quality of support scheme in MAC coating, and MAC broadcast mechanism. Jalali, Mohammad et al. proposed a fuzzy reputation process to discipline selfish conduct and inspire packet forwarding.. An ideal option is includes two important parts: The Ahead Supervisor and the Unclear Reputation Manager. The Ahead Supervisor in each node screens exactly how many acquired forwarding requests and the amount of packages which have been forwarded so far. Mohanty et al. discussed that Vehicular offer adhoc sites are a forthcoming skill that is developing traction in recent years. While doing aggregation reliability of the data can't be simply approved as well as episodes might be possible. In that report, they discussed as well as analyzed different data aggregation methods and their solutions. Asgari, Mojtaba et al. Proposed a new beaconless forwarding algorithm called CBBPF where data packages aren't broadcasted to the neighbors to prevent duplication. The simulative efficiency evaluation results in highway situations demonstrate that CBBPF runs effectively in regards to packet supply relation and average end-to-end delay. Amici, Raul et al. presented records to use some 
experiments to gauge the performance of a straightforward crisis project they in contrast to the simple random waypoint design to manage to measure how much the performance metrics are from this baseline. The outcomes display the possible outcomes of utilizing information dissemination via an opportunistic system that uses cab cabs being an information vector. Bali, Rasmeet et al. analyzed different difficulties and current solutions used for clustering in VANETs. The benefits are summarized the following: Firstly, an entire taxonomy on clustering in VANETs has been offered centered on different parameters. Based with this categorization, an comprehensive conversation is offered for each and every number of clustering including difficulties, current solutions and potential directions. Finally, a thorough examination of most of the current proposals in literature is offered regarding number of variables such as for instance as an example topology picked, extra infrastructure demands, road situation, node freedom, knowledge treated, and general way, width of the nodes, general rate, indication method, and indication overhead. The examination provided for different current proposals helps numerous people used in that domain to choose on the set of proposalsregarding its merits within the others. Eckhoff, Mark, and Christoph Sommer et al. introduced their state of the artwork in the simulation of vehicular communities, details when and how various, complicated real-life consequences should certanly be grabbed in a simulation product, and, finally, provides advice on the easiest way to acquire significant simulation results. Bazzi, Alessandro et al. discuss the device design and assess the mobile source saving that could be purchased in metropolitan cases through the deployment of WAVE/IEEE 802.11p units on the vehicles and roadside products, assessing the affect of the percentage of equipped vehicles, of the amount of deployed road area products, and of the followed redirecting protocol. Results, purchased via an integral simulation program taking both reasonable vehicular environments and instant network connection aspects under consideration, reveal that the deployment of several road area products and using reduced complexity redirecting standards benefits in a substantial reduced amount of mobile source occupation, also nearing $100 \%$ with a greater thickness of equipped vehicles. V.N.G.J. Soares et al. Discussed the difficulties related to redirecting and data dissemination in vehicular communities, and evaluates how delay-tolerant network methods may provide a option to deal with difficult vehicular connection environments. The section first introduces the many kinds of vehicular network applications. Then, alternatives planned in the literature and the study work that's been moved on it. Silva, Fabrício et al. planned and validated a macroscopic flexibility product that generates more reasonable features to the vehicular network evaluation process. The purchased benefits sign up to the study neighborhood by giving a far more reasonable macroscopic product that enhances many vehicular flexibility generators obtainable in the literature. Kumar, Neeraj et al. Planned a Understanding Automata-based Opportunistic Data Aggregation and Forwarding program for mindful generation in VANETs. Knowledge automata perform alone which are stationed to the closest Route Part Products to gather and forward the data from particular pieces along with mindful generation. Once information is aggregated, LA adaptively chooses the location for information transfer, on the cornerstone of the freshly explained full referred to as Opportunistic Aggregation and Forwarding.

\section{COMPARISON TABLE}

\begin{tabular}{|c|c|c|c|c|}
\hline Name of author & Title of the paper & Technique & Benefits & Limitations \\
\hline $\begin{array}{l}\text { Leng, Supeng, } \\
\text { Huirong Fu }\end{array}$ & $\begin{array}{l}\text { Medium access control } \\
\text { in vehicular ad hoc } \\
\text { networks }\end{array}$ & $\begin{array}{l}\text { VANET MAC } \\
\text { schemes }\end{array}$ & $\begin{array}{l}\text { To improve the connection } \\
\text { performance of VANETs, } \\
\text { MAC methods should be } \\
\text { created for optimizing } \\
\text { multichannel control and } \\
\text { allocation strategies, } \\
\text { improving the Quality of } \\
\text { Company (QoS) capability, } \\
\text { and overcoming the } \\
\text { concealed terminal problem, } \\
\text { transmitted surprise problem } \\
\text { and even ACK } \\
\text { (acknowledgment) explosion } \\
\text { problem. }\end{array}$ & NA \\
\hline Jalali & $\begin{array}{l}\text { A Fuzzy Reputation } \\
\text { System in Vehicular Ad } \\
\text { hoc Networks. }\end{array}$ & $\begin{array}{l}\text { fuzzy reputation } \\
\text { system }\end{array}$ & $\begin{array}{l}\text { To improve the network } \\
\text { performance }\end{array}$ & NA \\
\hline
\end{tabular}




\begin{tabular}{|c|c|c|c|c|}
\hline Mondragon & $\begin{array}{l}\text { Intelligent transport } \\
\text { systems in multimodal } \\
\text { logistics: A case of role } \\
\text { and contribution through } \\
\text { wireless vehicular } \\
\text { networks in a sea port } \\
\text { location. }\end{array}$ & $\begin{array}{l}\text { Intelligent } \\
\text { Transport } \\
\text { Systems }\end{array}$ & $\begin{array}{l}\text { To enhance the degrees of } \\
\text { awareness, responsiveness } \\
\text { and effectiveness in present } \\
\text { chains counting in } \\
\text { multimodal transfer } \\
\text { operations. }\end{array}$ & NA \\
\hline Mohanty & $\begin{array}{l}\text { Secure data aggregation } \\
\text { in vehicular-adhoc } \\
\text { networks: A survey }\end{array}$ & Data aggregation & $\begin{array}{l}\text { The goal of data aggregation } \\
\text { is to mix the } \\
\text { communications and } \\
\text { disseminate that in greater } \\
\text { region. }\end{array}$ & $\begin{array}{l}\text { Because of the restricted } \\
\text { bandwidth of instant } \\
\text { communication medium, } \\
\text { scalability is just a } \\
\text { important problem. }\end{array}$ \\
\hline Asgari & $\begin{array}{l}\text { Reliable Contention- } \\
\text { based Beaconless Packet } \\
\text { Forwarding Algorithm } \\
\text { for VANET Streets }\end{array}$ & $\begin{array}{l}\text { beaconless } \\
\text { forwarding } \\
\text { algorithm called } \\
\text { CBBPF }\end{array}$ & $\begin{array}{l}\text { These methods contributes to } \\
\text { supply duplications in both } \\
\text { forwarding area and the } \\
\text { location node and therefore } \\
\text { advances the system } \\
\text { overhead and wastes } \\
\text { available bandwidth. }\end{array}$ & NA \\
\hline Harrabi & $\begin{array}{l}\text { A Multi-agent Approach } \\
\text { for Routing on Vehicular } \\
\text { Ad-Hoc Networks }\end{array}$ & $\begin{array}{l}\text { a multi-agent } \\
\text { system approach }\end{array}$ & $\begin{array}{l}\text { To enhance the performance } \\
\text { of Vehicular ad-hoc network } \\
\text { routing. }\end{array}$ & $\begin{array}{l}\text { The key problem with } \\
\text { your methods in } \\
\text { VANETs environments } \\
\text { is their option } \\
\text { instability. }\end{array}$ \\
\hline Yan & $\begin{array}{l}\text { A Novel Vehicular } \\
\text { Information Network } \\
\text { Architecture based on } \\
\text { Named Data Networking } \\
\text { (NDN }\end{array}$ & $\begin{array}{l}\text { named data } \\
\text { networking } \\
(\mathrm{NDN})\end{array}$ & $\begin{array}{l}\text { To boost content labeling, } \\
\text { addressing, knowledge } \\
\text { aggregation, and flexibility } \\
\text { for IVC in the vehicular } \\
\text { information network. }\end{array}$ & NA \\
\hline Kumar & $\begin{array}{l}\text { Learning automata-based } \\
\text { opportunistic data } \\
\text { aggregation and } \\
\text { forwarding scheme for } \\
\text { alert generation in } \\
\text { vehicular ad hoc } \\
\text { networks }\end{array}$ & $\begin{array}{l}\text { Learning } \\
\text { Automata-based } \\
\text { Opportunistic } \\
\text { Data Aggregation } \\
\text { and Forwarding } \\
\text { (LAODAF) } \\
\text { scheme }\end{array}$ & $\begin{array}{l}\text { It changes their activity } \\
\text { chance vector and learning } \\
\text { charge on the basis of the } \\
\text { prices of OAF. }\end{array}$ & $\begin{array}{l}\text { It finds the how and } \\
\text { where in fact the } \\
\text { collected data is to be } \\
\text { given }\end{array}$ \\
\hline Slavik & $\begin{array}{l}\text { Analysis and evaluation } \\
\text { of distance-to-mean } \\
\text { broadcast method for } \\
\text { VANET }\end{array}$ & $\begin{array}{l}\text { distance-to-mean } \\
\text { (DTM) }\end{array}$ & $\begin{array}{l}\text { It exhibit a higher amount of } \\
\text { reach ability across a wide } \\
\text { selection of situations }\end{array}$ & NA \\
\hline
\end{tabular}

\section{CONCLUSION}

To resolve the issues of IOT, this work will propose a novel technique that enhances the NDN model. To efficiently score location based forwarding, data aggregation as well as distributed mobility in management using compressive sensing will be utilized. Also this work will focuses on the energy issues of NDN for VANETs. The effect of environmental conditions on VANETs will also be considered in this work.

\section{REFERENCES}

[1] Leng, Supeng, Huirong Fu, Qing Wang, and Yan Zhang. "Medium access control in vehicular ad hoc networks." Wireless Communications and Mobile Computing 11, no. 7 (2011): 796-812.

[2] Jalali, Mohammad, and Nasser Ghasem Aghaee. "A Fuzzy Reputation System in Vehicular Ad hoc Networks." Procedia Computer Science 5 (2011): 951956. 
[3] Mondragon, Adrian E. Coronado, Chandra S. Lalwani, Etienne S. Coronado Mondragon, Christian E. Coronado Mondragon, and Kulwant S. Pawar. "Intelligent transport systems in multimodal logistics: A case of role and contribution through wireless vehicular networks in a sea port location."International Journal of Production Economics 137, no. 1 (2012): 165-175.

[4] Mohanty, Sagarika, and Debasish Jena. "Secure data aggregation in vehicular-adhoc networks: A survey." Procedia Technology 6 (2012): 922-929.

[5] Asgari, Mojtaba, Mahamod Ismail, and Raed Alsaqour. "Reliable Contention-based Beaconless Packet Forwarding Algorithm for VANET Streets." Procedia Technology 11 (2013): 1011-1017.

[6] Harrabi, Samira, Walid Chainbi, and Khaled Ghedira. "A Multi-agent Approach for Routing on Vehicular Ad-Hoc Networks." Procedia Computer Science 19 (2013): 578585 .

[7] Amici, Raul, Marco Bonola, Lorenzo Bracciale, Antonello Rabuffi, Pierpaolo Loreti, and Giuseppe Bianchi. "Performance Assessment of an Epidemic Protocol in VANET Using Real Traces." Procedia Computer Science 40 (2014): 92-99.

[8] Bali, Rasmeet S., Neeraj Kumar, and Joel JPC Rodrigues. "Clustering in vehicular ad hoc networks: taxonomy, challenges and solutions." Vehicular communications 1 , no. 3 (2014): 134-152.

[9] Yan, Zhiwei, Sherali Zeadally, and Young-Jin Park. "A Novel Vehicular Information Network Architecture based on Named Data Networking (NDN).", IEEE Journal I nternet of Things, Vol1 , no. 6, pp: 525 - 532, 2014.
[10] Bravo-Torres, Jack F., Martín López-Nores, Yolanda Blanco-Fernández, José J. Pazos-Arias, and Esteban F. Ordóñez-Morales. "VaNetLayer: A virtualization layer supporting access to web contents from within vehicular networks."Journal of Computational Science (2014).

[11] Kumar, Neeraj, Naveen Chilamkurti, and Joel JPC Rodrigues. "Learning automata-based opportunistic data aggregation and forwarding scheme for alert generation in vehicular ad hoc networks." Computer Communications 39 (2014): 22-32.

[12] Slavik, Michael, Imad Mahgoub, and Mohammed M. Alwakeel. "Analysis and evaluation of distance-to-mean broadcast method for VANET." Journal of King Saud University-Computer and Information Sciences 26, no. 1 (2014): 153-160.

[13] Eckhoff, David, and Christoph Sommer. "Simulative performance evaluation of vehicular networks." (2015): 255-274.

[14] Bazzi, Alessandro, Barbara M. Masini, Alberto Zanella, and Gianni Pasolini. "IEEE $802.11 \mathrm{p}$ for cellular offloading in vehicular sensor networks." Computer Communications 60 (2015): 97-108.

[15] ++V.N.G.J. Soares, J.J.P.C. Rodrigues, “ Vehicular delay-tolerant networks (DTNs)", Advances in DelayTolerant Networks (DTNs), Pages 61-80, 2015.

[16] Silva, Fabrício A., Azzedine Boukerche, Thais RMB Silva, Linnyer B. Ruiz, and Antonio AF Loureiro. "A novel macroscopic mobility model for vehicular networks." Computer Networks (2015) 